\title{
A novel animal model to explore the whole- organism response to 21-hydroxylase deficiency
}

UNIVERSITYOF BIRMINGHAM
Andreas Zaucker, Tulay Guran, Nazia Thakur, Angela Taylor, Aliesha Griffin \& Nils Krone University of Birmingham, Birmingham, West Midlands, UK.

\section{Introduction}

Challenges in studying CAH (Congenital Adrenal Hyperplasia):

- Comorbidities in CAH patients on long-term treatment not fully understood

- In vitro studies on CAH mutations do

not always correlate with patient phenotypes

-210H deficiency (210HD) difficult

to study in mice -> mutants are not viable

- Incomplete understanding of

systemic consequences of $210 \mathrm{HD}$

21-hydroxylase

(210H, CYP21A2)

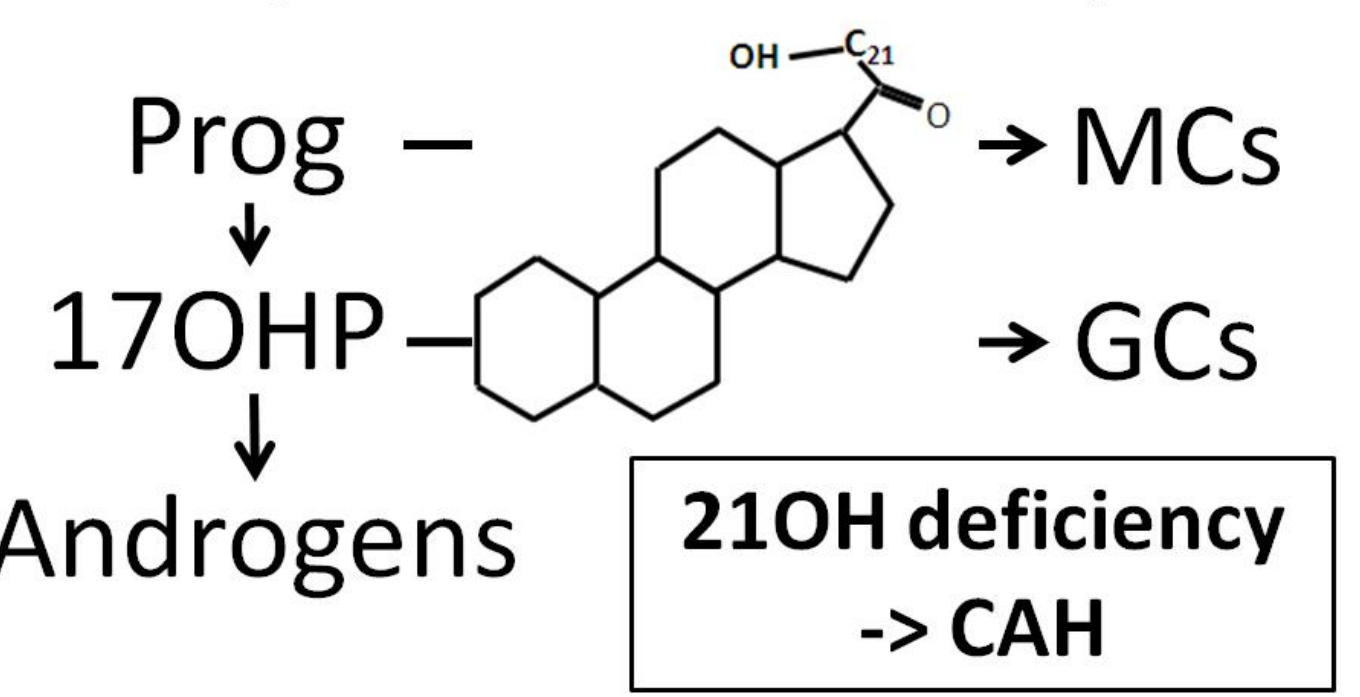

Need for novel in vivo models for $\mathbf{2 1 0 H}$ deficiency Aim:

To establish a novel in vivo model for $210 H D$ using zebrafish

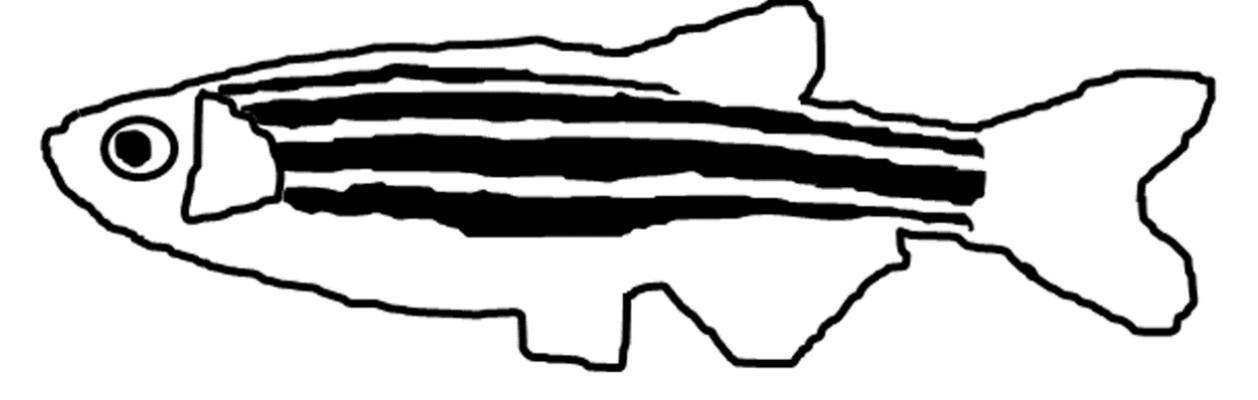

cyp21a2 mutants
Conclusions:

Zebrafish cyp21a2 mutants are a promising model to study 210HD

1. 21-hydroxylase is conserved in zebrafish

2. Zebrafish cyp21a2 mutants have impaired GC signalling

3. Zebrafish cyp21a2 mutants have dysregulated HPA axis

\section{Results}

\section{5 days zebrafish cyp21a2 mutants} have enlarged interrenals (adrenals)

ISH against cyp17a2 (interrenal, blue arrow) in cyp21a2 mutants and siblings

top views

side views

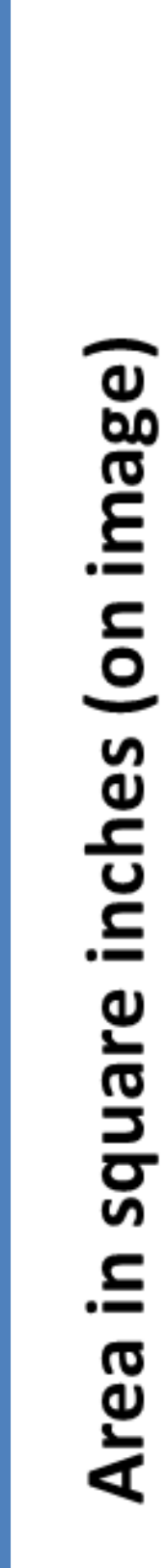

Cyp21a2 mutants display pigmentation phenotype (VBA, GC deficiency)

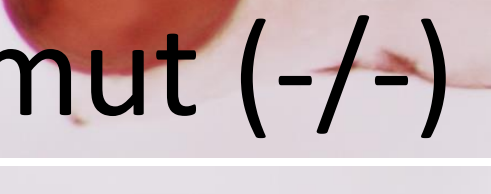

wt $(+/+)$

het $(+/-)$

mut $(-/-)$

Size of interrenal gland in $\mathbf{5}$ days larvae stained for cyp17a2 mRNA (area \pm sd)
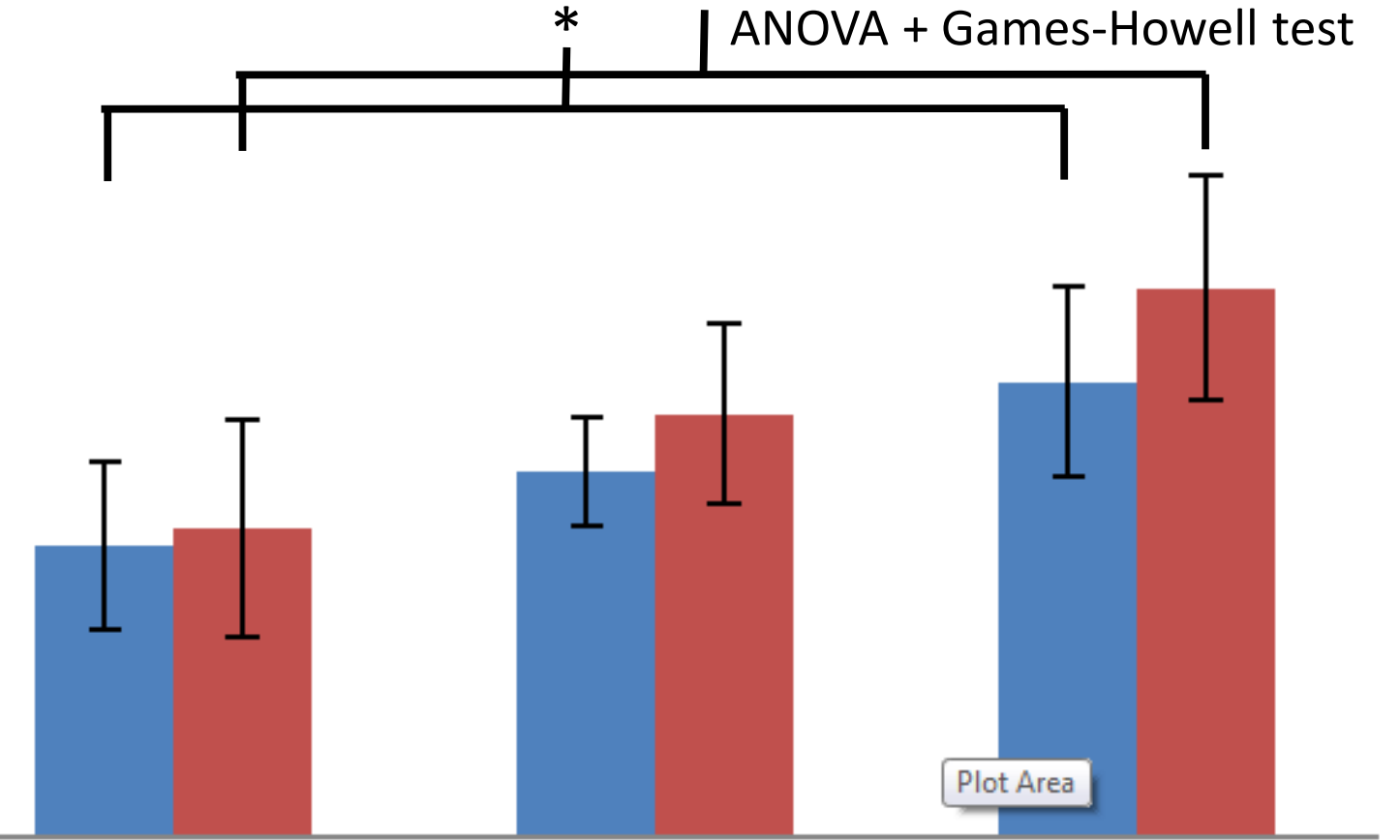

- cyp17a2 positive are

in side view images

cyp17a2 positive are in top view images

wtt/+ (n=4) het $+-(n=7)$ mut- $/(n=8)$ Genotype regarding cyp21a2

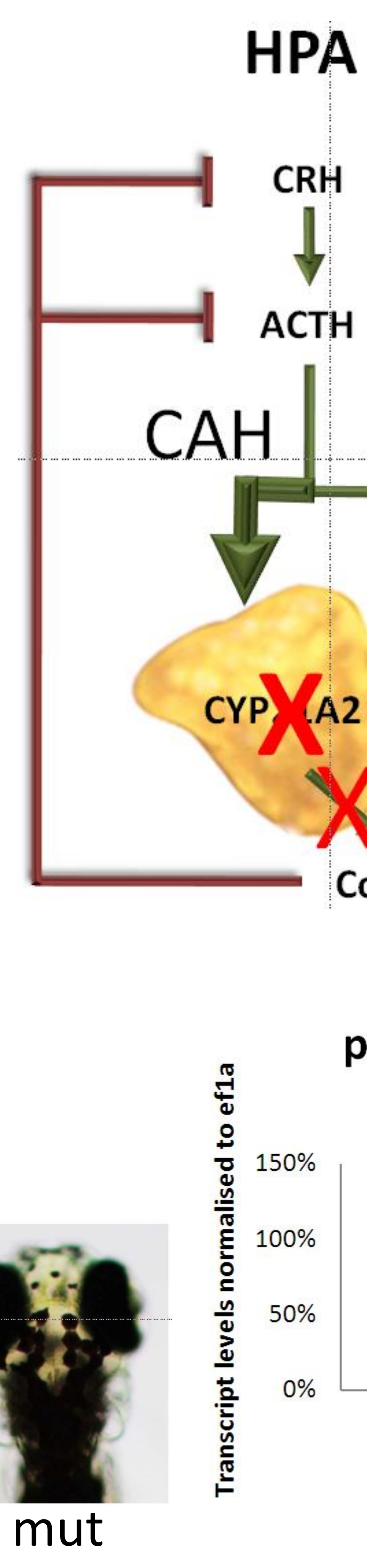

HPA axis

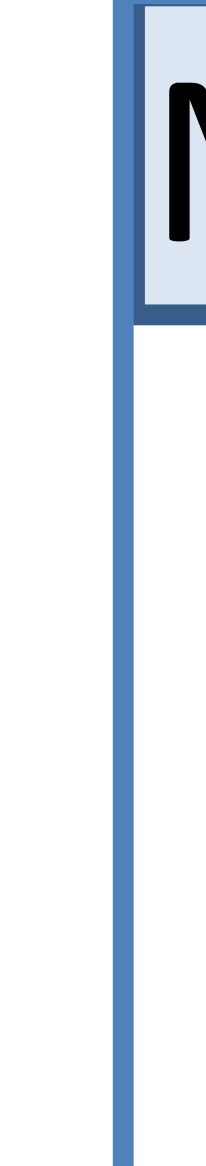

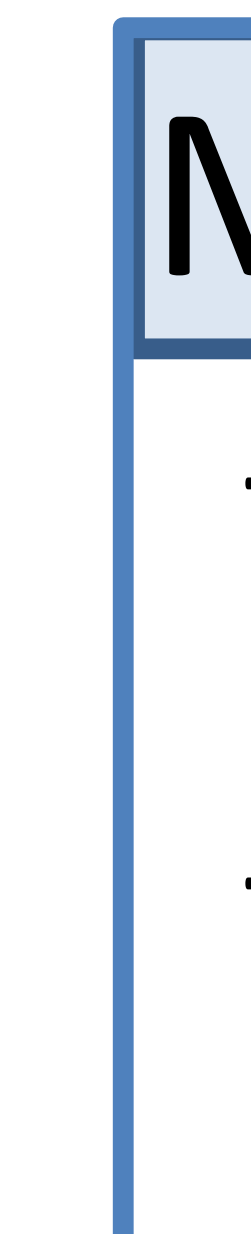

Material and Methods

Three cyp21a2 mutant lines were generated by TALEN mediated mutagenesis

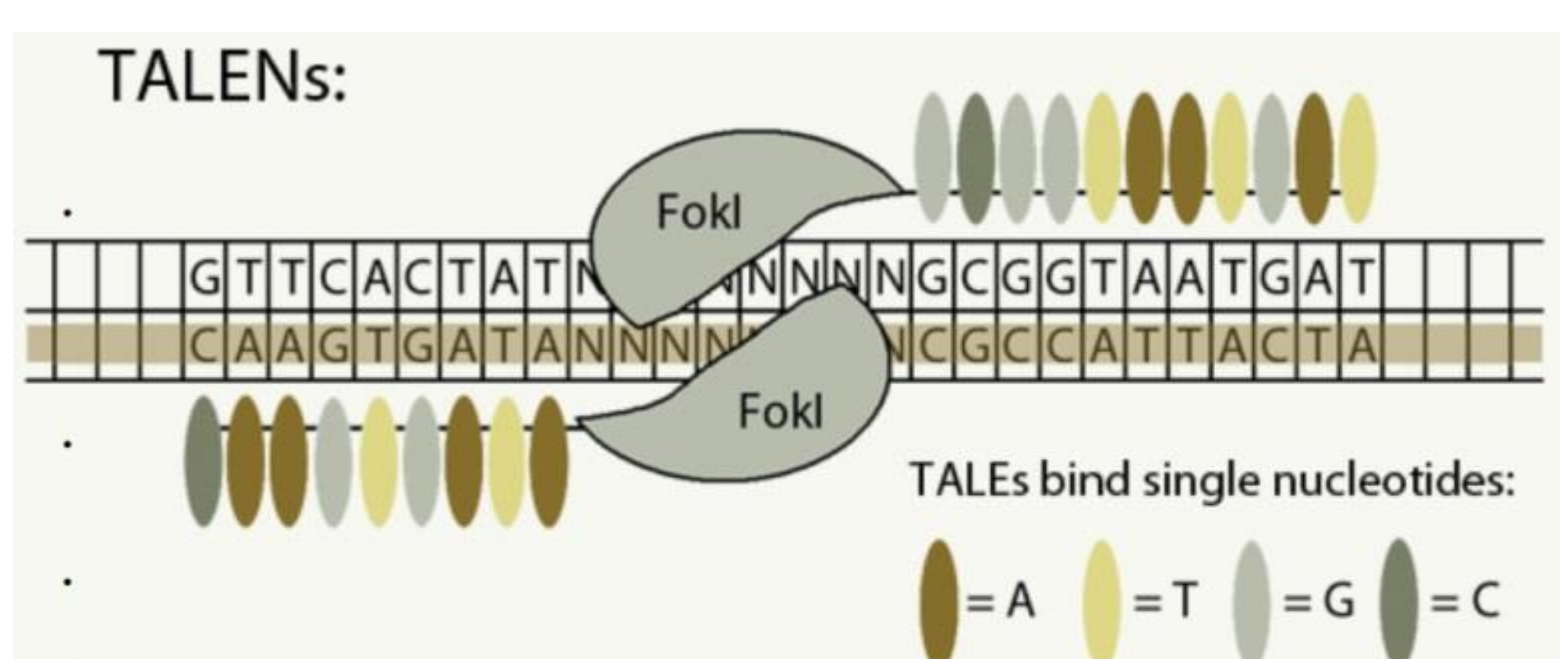

$$
\text { cyp }
$$

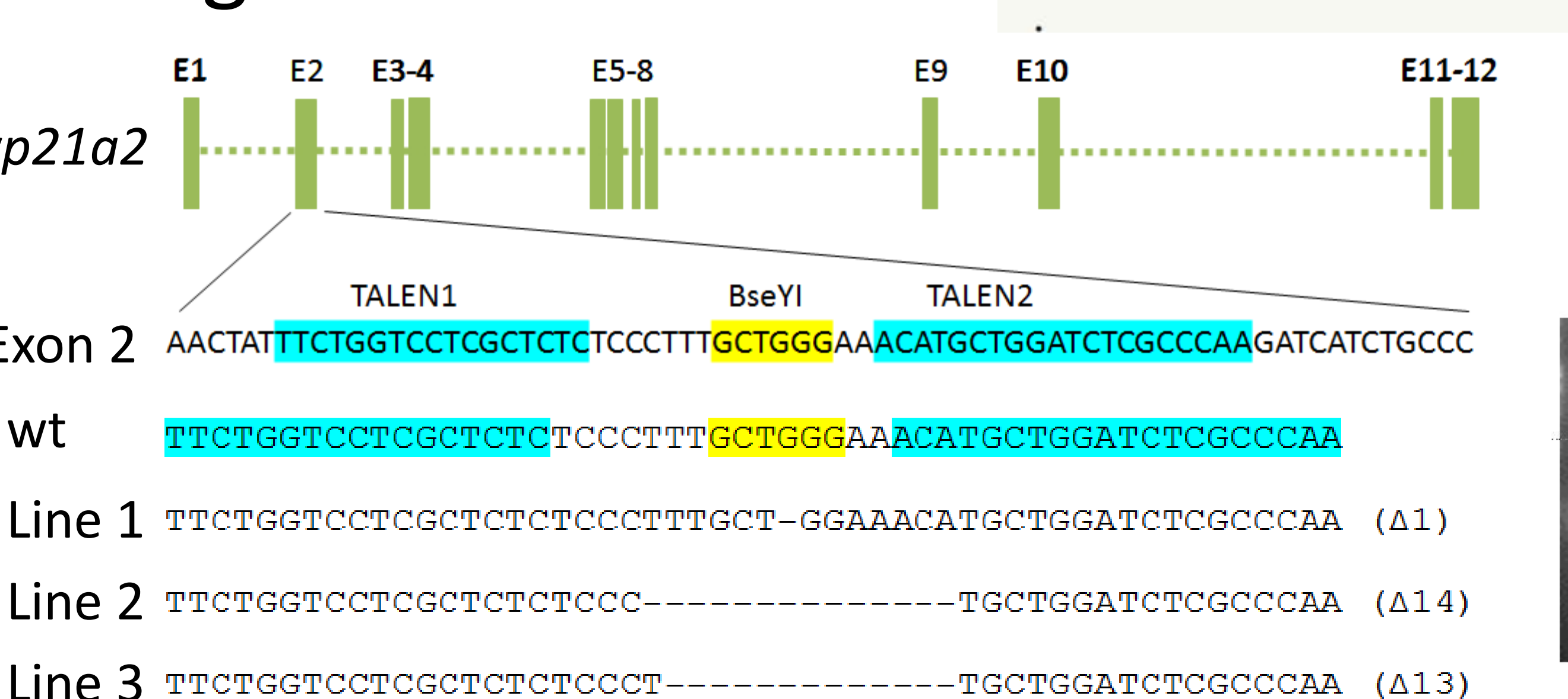

Genotyping by BseYl-assay
Hypothalamus

Pituitary

Healthy

Increased expression of ACTH precursor pomca in cyp21a2 mutants

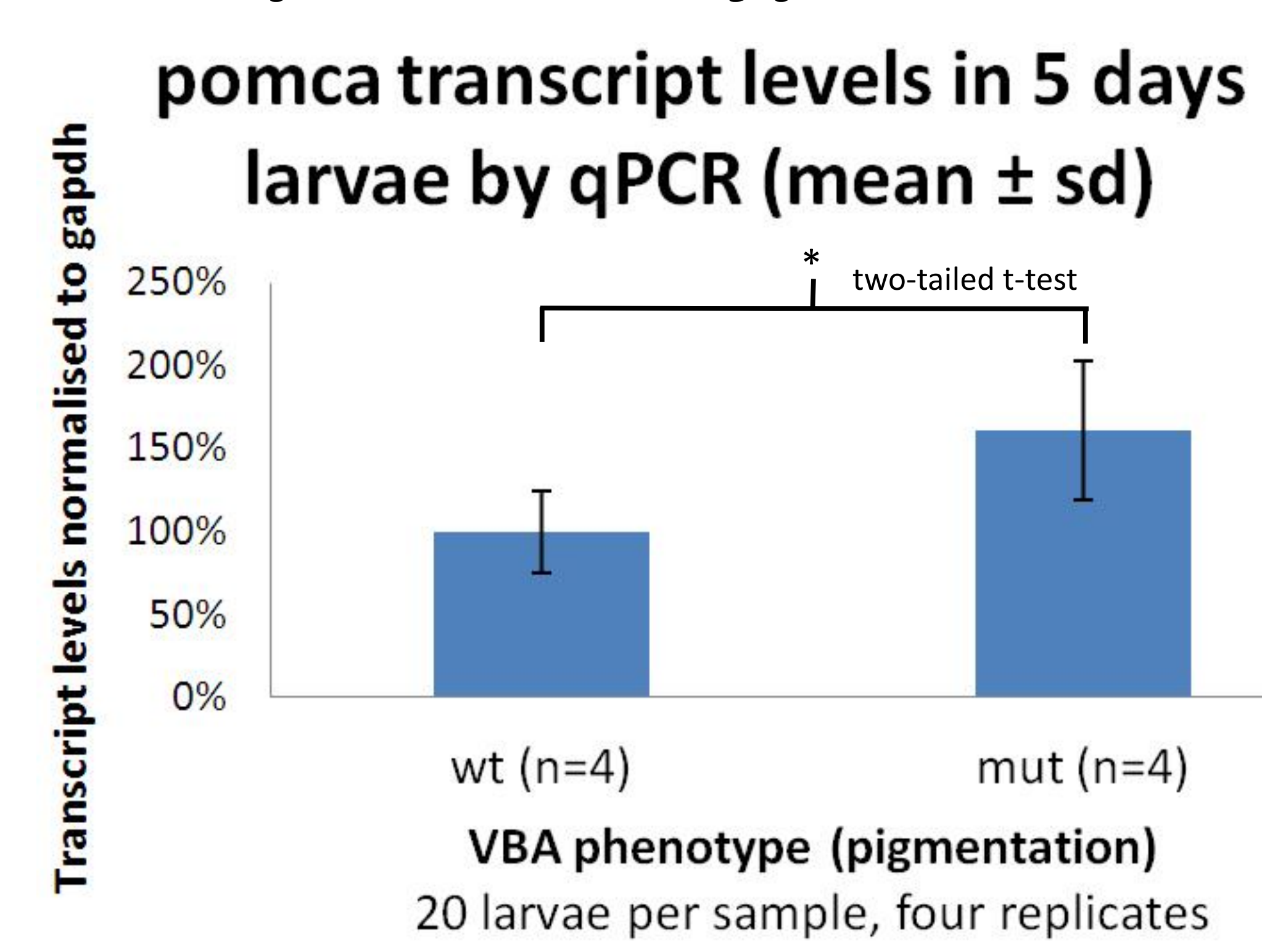

Reduced expression of GR targets fkbp5 and pck1 in cyp21a2 mutants
Cortisol (GCs)

pck1 transcript levels in $\mathbf{5}$ days larvae by qPCR (mean $\pm s d$ )

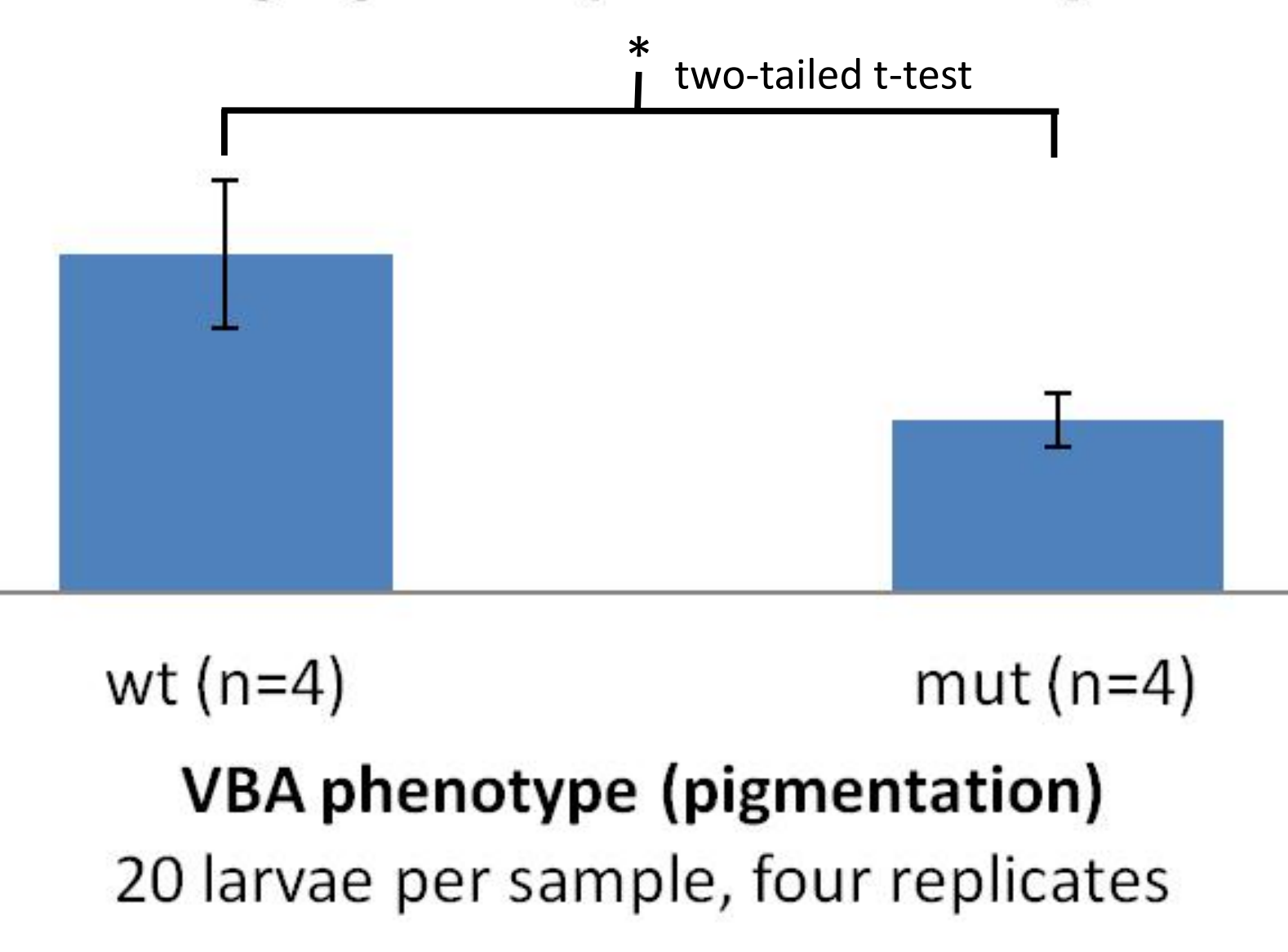

fkbp 5 transcript levels in 5 days

larvae by qPCR (mean $\pm \mathrm{sd}$ )

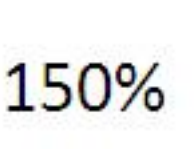

$150 \%$

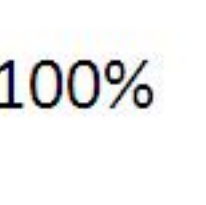

$50 \%$

$0 \%$

wt $(n=4)$

mut $(n=4)$

VBA phenotype (pigmentation) 20 larvae per sample, four replicates

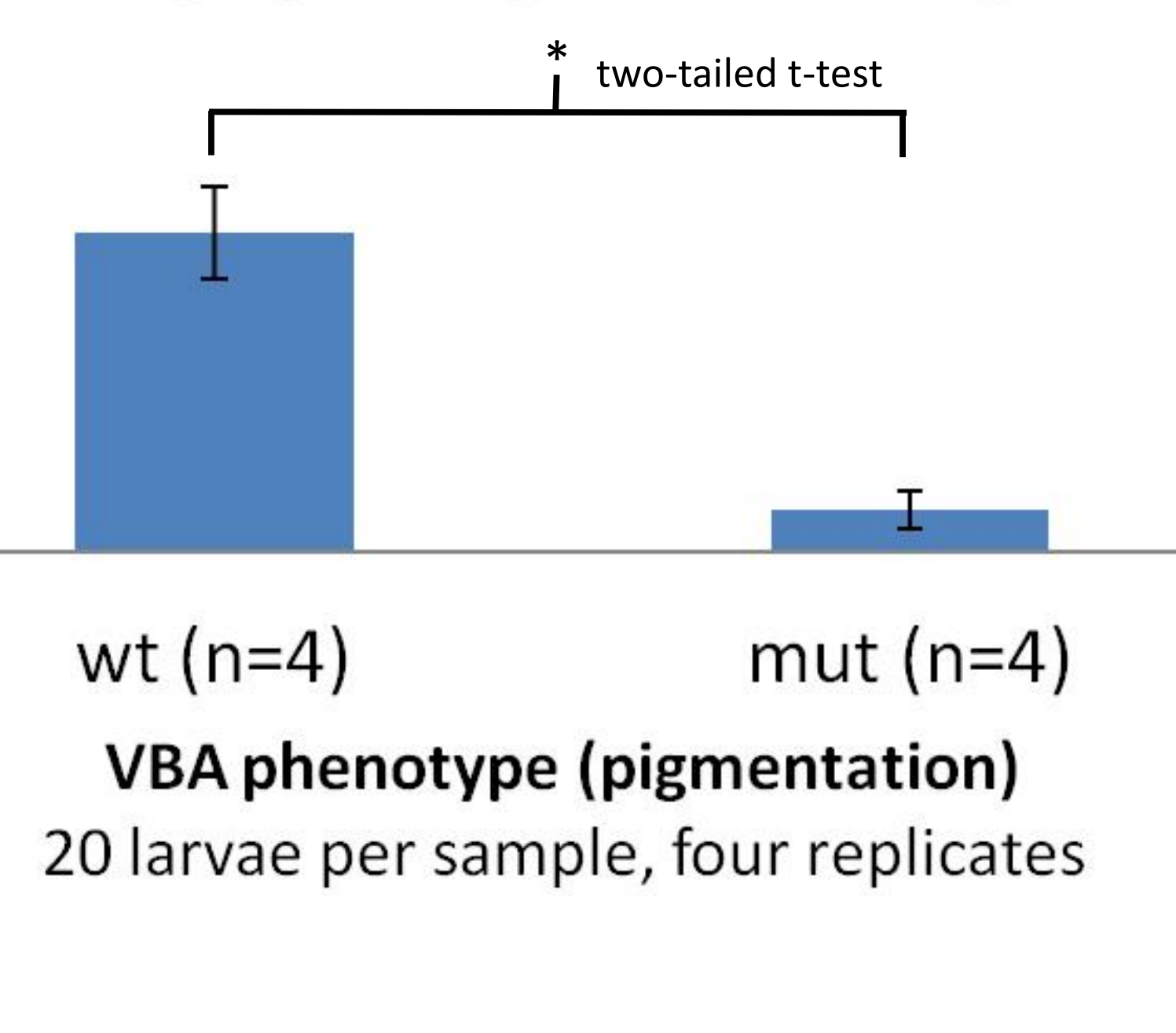

\section{Acknowledgments}

Society for Endocrinology

Early Career Grant to Andreas Zaucker
I F C A H平 In te rn ation al fund
Congenital Adrenal Hyperplasia

Project grant to Nils Krone 